\title{
Los jagüeyes comunitarios como un sistema ambiental antrópico y la importancia de su gestión
}

\author{
The community cattle ponds as antropic environmental system and the \\ importance of its management
}

De La Ossa-Lacayo, Alejandro M.Sc, Herrera-Betín, Jorge Mario² Esp.

${ }^{1}$ Selvagua S.A.S. Sincelejo, Sucre. Colombia. ${ }^{2}$ Alcaldía Municipal de Sincelejo, Sucre, Colombia.

\section{Key words:}

Cattle ponds; environmental system; management; Caribbean; Colombia.

\begin{abstract}
The present revision exposes important aspects that it should be assisted for the appropriate administration of the regional cattle ponds, it analyzes concepts related with the environmental management and its dimension, hydric administration and its focuses, the basin and its conceptual delimitation, the different study levels that can be observed for the water resource administration, climatic change and their impact on the hydrological cycle, to conclude with the importance that the cattle ponds possess taken into account its use and social importance, leaving established the value that these antropic environmental systems possess in the local conservation and their cultural value.
\end{abstract}

\section{Palabras Clave:}

\section{Jagüeyes;} sistema ambiental; gestión;

Caribe;

Colombia.

\section{Resumen}

El presente trabajo de revisión expone aspectos importantes que se deben tener en cuenta para la adecuada gestión de los jagüeyes regionales, analiza conceptos relacionados con la gestión ambiental y su dimensión, gestión hídrica y sus enfoques, la cuenca y su delimitación conceptual, los diferentes niveles de estudio que se pueden tener respecto de la administración del recurso agua, cambio climático y su impacto sobre el componte hidrológico, para finalizar con la importancia que poseen los jagüeyes en términos de uso y aprovechamiento social, dejando plasmado el valor que estos sistemas ambientales antrópicos poseen en la conservación local y su valor cultural. 


\section{Introducción}

Actualmente el agotamiento del recurso hídrico es una de las mayores preocupaciones de la comunidad mundial. Los ambientalistas no han sido ajenos a esta problemática, es por esto que en los últimos años, desde la economía ambiental se han desarrollado marcos teóricos y metodológicos, para investigar y buscar diferentes soluciones a la problemática en referencia (ENVIRONMENTAL RESOURSES MANAGEMENT Y DEPARTAMENT FOR INTERNATIONAL DEVELOPMENT,1997).

ENVIRONMENTAL RESOURSES MANAGEMENT Y DEPARTAMENT FOR INTERNATIONAL DEVELOPMENT (1997) indica, en cuanto al recurso hídrico, que se tienen como primordiales, los valores de uso: se refiere a los valores que la gente obtiene por la utilización del recurso de la cuenca hidrográfica, puede ser directo en relación con el ecosistema, vincula el consumo de agua, la pesca y leña, por ejemplo. Puede ser indirecto, cuando hace referencia a valores obtenidos recreación y turismo, entre otros. Por otra lado, están los valores de no uso, aquellos que se mantienen a pesar que los usuarios no utilicen un recurso, es posible que este sea valioso para ellos. Este valor tiene dos formas; la primera es un valor de legado, es decir el deseo de que las futuras generaciones gocen de una cierta dotación de recursos naturales; la segunda es el valor de existencia, que se asocia simplemente con el conocimiento de existencia del recurso.

La valoración económica del medio ambiente, incluye un conjunto de métodos cuantitativos por medio de los cuales se intenta asignar valor económico a los bienes, servicios y atributos proporcionados por los recursos naturales y ambientales, independientemente de que estos tengan o no mercado. También, se le considera como una herramienta que permite medir bajo una unidad común, las ganancias tangibles que tiene para la sociedad conservar, proteger, restaurar, o recuperar el medio ambiente y los recursos naturales; o por el contrario, los costos de la contaminación, la sobreexplotación y el deterioro de los mismos. (CASTIBLANCO, 2005).

Los ecosistemas de aguas continentales cubren una pequeña porción de la superficie terrestre (KALFF, 2001) y ocupan un rango que oscila entre 2-2,8 $\times 106$ $\mathrm{km}^{2}$ (MEYBECK, 1995; KALFF, 2001; SHIKLOMANOV y RODDA, 2003). Constituye solo entre el 1,3 y 1,8 de la superficie no oceánica, numéricamente dominada por pequeños sistemas (SCHUILING, 1977; WETZEL, 1990; MEYBECK, 1995). En cuanto a la cobertura, se estima en 4,2 millones de $\mathrm{km}^{2}$ con una representación de 304 millones de lagos (DOWNIG et al., 2006).
Según CERVANTES (1994) los jagüeyes son lagunas con aparente similitud con los lagos, su existencia puede corresponder a cualquier origen, drenaje y dimensiones. Igualmente indica que permanecen relativamente estancados y son inestables, con variaciones en el nivel de agua; pueden ser temporales o permanentes, dependiendo del régimen pluvial.

DECLERK et al. (2006) indican que existe una relación positiva entre el número de jagüeyes y la biodiversidad de un área dada; las áreas acuáticas restringidas como los jagüeyes juegan un importante papel en la conservación, contribuyen significativamente a la biodiversidad regional, en especial cuando poseen como habitantes especies raras, endémicas o únicas, por esta razón la creación de nuevas unidades viene siendo usada como estrategia ampliamente practicada en muchos países. CHARÁ et al. (2008) señalan que permiten, en efecto, el establecimiento y conservación de especies vegetales y organismos animales diversos, proveen alimento y refugio, tanto para seres vivos propios del agua como de aquellos que se asocian al sistema por las facilidades ofrecidas. Igualmente son fuente de agua para las comunidades rurales asentadas en su área de influencia, concordando con BOTERO et al. (2009)

Según BALLUT-DAJUD y MONROY-PINEDA (2015) en el municipio de Sincelejo, departamento de Sucre, Colombia se tienen 300 unidades, La representación areal de los jagüeyes corresponde al $0,43 \%$ del territorio municipal, lo que equivale en promedio a un jagüey por cada 95 ha. En cuanto a sus áreas se determina que $89,3 \%$ están entre 0,1 y 1 ha, $5,7 \%$ entre 1,1 y 2 ha, $2,7 \%$ entre 2,1 y 3 ha, $1 \%$ entre 3,1 y 4 ha, $1,3 \%$ mayores $4,1 \mathrm{ha}$. Lo cual indica la dependencia de este tipo de modelo de almacenamiento hídrico que tienen las comunidades rurales del municipio de Sincelejo y la importancia de su estudio.

\section{Gestión ambiental}

De manera general, la gestión ambiental se concibe de diversas maneras, coincidentes todas en el aspecto fundamental de protección ambiental. Se tiene, que COLBY (1990) considera a la gestión ambiental como el campo que busca equilibrar la demanda de recursos naturales de la Tierra con la capacidad del ambiente; la gestión ambiental debe responder a esas demandas en una base sustentable; conciliando las actividades humanas y el medio ambiente a través de instrumentos que estimulen y hagan viable esa tarea, lo que presupone la modificación del comportamiento del ser humano en relación con la naturaleza, debido a la actual situación de degradación del ambiente. Por su parte, BASTERRECHEA et al. (1996) indican que la gestión ambiental debe entenderse como el conjunto 
de acciones gerenciales de tipo técnico, administrativo, legal, financiero y económico, dándole una visión empresarial.

RODRÍGUEZ y ESPINOZA (2002) definen la gestión ambiental como un proceso permanente y de aproximaciones sucesivas en el cual diversos actores públicos, privados y de la sociedad civil desarrollan un conjunto de esfuerzos específicos con el propósito de preservar, restaurar, conservar y utilizar de manera sustentable el ambiente. Siendo complementada desde un punto de vista legal por ALBÁN et al. (2003) quienes la asocian al conjunto de políticas, normas y acciones que desarrollan las instituciones públicas, pero también las comunidades y los individuos, con relación al manejo de los recursos naturales, sean éstos renovables o no renovables.

RODRÍGUEZ y ESPINOZA (2002) consideran a la gestión ambiental como una vía de interpretación de los problemas ambientales, que se puede abordar desde diversas perspectivas y con diferentes escalas: local o global; puede ser analizada desde los distintos niveles de gobierno: nacional, departamental o municipal; desde diferentes ámbitos territoriales: global, regional, local, urbano, rural o puntual. No obstante, es importante tener presente que dentro de la gestión ambiental está implícita una selección estratégica de ciertos procesos, que obliga a jerarquizar el grado de incidencia y de sus efectos, determinantes y condicionantes sobre una problemática concreta, tal como señalan GALLOPÍN $(1985,2000)$ y MONTES y LEFF (2000).

\section{Gestión hídrica y sus enfoques}

La gestión hídrica, como parte de la gestión ambiental, puede abordarse desde dos enfoques: extractivo e integral. En tal sentido, las percepciones de los diferentes agentes y de la comunidad en general son esenciales para la gestión y uso del agua e incluso para la estabilidad sociopolítica y territorial. En esencia son los actores quienes determinan en última instancia cuál es el enfoque que determina el uso del recurso (DOUROJEANNI, 2002).

Extractivamente se considera este recurso como un producto a ser obtenido sin tener en cuenta el impacto de su uso irracional o de su utilización no sostenible. Cuando esto sucede se enfrentan decisiones de orden político que se contraponen, en muchos casos, a consideraciones técnicas e incluso ambientales y que pueden favorecer con exclusividad aspectos económicos y dejar de lado situaciones sociales que suelen ser críticas en función del tiempo y del uso del recurso (DANILO y DÍAZ, 2000). El enfoque extractivo se fundamenta ampliamente en el establecimiento de regulaciones jurídicas, las cuales, la mayoría de las veces son muy generales y no responden a la especificidad que demanda la gestión del agua (ALBUQUERQUE, 2000).

El enfoque integral privilegia bajo perspectivas diversas, la concurrencia de distintos y variados modos de abordar la gestión, siendo incluyente con las diversas disciplinas que allí pueden concurrir. En tal sentido, por ejemplo, PATRICK (1994) conceptúa que la crisis del agua se podría reducir si los agentes estatales y los gobiernos trabajaran usando el conocimiento de diversos campos de la ciencia. El enfoque integral considera que para que exista una adecuada gestión hídrica y se tenga inclusión social se deben tener en cuenta aspectos económicos, políticos y culturales, así como las instituciones, los conflictos y los intereses de los distintos actores (GRIGG, 1996; QUINTANA y FUNTOWICZ, 2000).

Según OPS/OMS (2001) el enfoque integral necesario para hacer gestión del agua, requiere saber manejar la oferta de este recurso de manera sostenible en el tiempo, con el fin de entender los requerimientos en términos de cantidad, calidad y distribución espacial, al tiempo que se deben considerar todos los elementos de índole natural y social que están presentes en el proceso.

\section{La cuenca como unidad conceptual}

CEPAL (2001) señala que la cuenca es la unidad óptima de gestión territorial para la planificación y administración de los recursos hídricos. Sin embargo, también aduce que las jurisdicciones político-administrativas no coinciden con los límites territoriales de las cuencas. Más aún, algunas de las decisiones que afectan al ciclo hidrológico, el uso del agua y a los habitantes de una cuenca se toman sin considerarla como un sistema integrado. Lo anterior también es considerado por la GWP (2000) cuando indica que el manejo de los recursos hídricos y el suelo debe garantizar que se mantengan los ecosistemas de la cuenca.

Con el fin de dejar en clatro el concepto de cuenca, se tiene que en la reunión anual de la Asociación Mundial de Hidrología de Cuencas, celebrada en 1992, se estableció que la unidad fundamental para el manejo de los recursos agua, suelo y vegetación era la cuenca (OROPEZA, 1999). Pero BASTERRECHEA et al. (1996) van más allá y conceptúan que las posibilidades de diferenciación espacial y de integración conceptual de la cuenca hidrográfica hacen de ella un marco geográfico propicio para entender los impactos humanos y naturales. La definen como el espacio apropiado para planificar e implementar las actividades y medidas de corrección de impactos que son producto del uso y manejo de los recursos. 
En contraposición MELVILLE (2000), reconoce que la cuenca hidrográfica es un concepto útil para planificar el aprovechamiento del agua al servicio de diversas necesidades humanas. No obstante, indica que hay que admitir que esta conceptualización no corresponde en todas las circunstancias geográficas e históricas con una viabilidad práctica. Es frecuente que entre territorios se presente variedad de identidades sociales y de rasgos culturales asociados a los usos del agua, lo que hace que se deba interpretar en sentido diferente por las circunstancias que los separan.

En la mayoría de los países de América Latina, Colombia no es la excepción, se acepta el concepto de cuenca como espacio físico idóneo para la gestión integral de los recursos hídricos (DOUROJEANNI, 2002). También es evidente que en América Latina existe un largo historial de gestión del agua a nivel de cuencas; se tiene una trayectoria variada con distintos niveles de continuidad y de cobertura, llegando a tener variaciones significativas interterritoriales, con grandes rasgos de informalidad en muchos casos y en ocasiones con apropiaciones culturales muy particulares o con cobertura social inapropiada (AGUILAR-ROBLEDO, 1995; CASTELÁN 2000; MARTÍNEZ, 1999; MELVILLE, 1996, 2000). La experiencia en América Latina y de otros países en el proceso de gestión integral es incipiente; se puede decir que se practica en muy pocas cuencas y solo en algunos países (SANTACRUZ-DE LEÓN, 2007).

(SANTACRUZ-DE LEÓN, 2007) concluye, que dentro de las definiciones de la cuenca hidrográfica no se tiene claridad respecto de que la cuenca hidrográfica es en sí misma un sistema hídrico complejo en donde convergen el sistema biofísico y el sistema socioeconómico; se acepta que entre los dos sistemas se generan relaciones e interdependencias. No obstante, se deja el concepto de interrelación dinámica en el campo teórico y no se explica cómo abordar la realidad de sus relaciones y sus sinergias. Igualmente, no se dejan claros los límites, porque no se trata solamente de límites físicos, ya que los límites se pueden interpretar como barreras permeables a través de las cuales se establecen flujos en diferentes direcciones, con diversas magnitudes y variados impactos.

Sin contar que dentro de un sistema y más aún si es complejo, existen unidades que lo conforman y que también pueden ser complejas, se les reconoce como subsistemas, que interactúan entre sí y las interrelaciones que tienen entre ellas constituyen las condiciones que determinan límites para cada subsistema (GARCÍA, 1986; MIRAMONTES, 1999).

\section{Niveles del sistema en estudio}

GARCÍA (1986) describe los cambios que tienen lugar en un sistema, haciendo una distinción entre los niveles de procesos y niveles de análisis; diferencia tres niveles de procesos: básico, permite analizar el efecto local sobre el medio físico o la sociedad que lo habita y lo usufructúa. El segundo, inmerso en la transformación, es un nivel que determina los procesos del primero y tiene que ver con las modificaciones en el sistema productivo por la implantación agrícola, extractivismo o uso del suelo y factores comerciales. El tercero, determina las modificaciones del segundo nivel, allí se tienen las políticas nacionales de desarrollo y las reglas del mercado internacional y sus exigencias globales.

Teniendo en cuenta lo antes señalado, para realizar el estudio del sistema ambiental hídrico y sus complejidades se debe hacer el reconocimiento de situaciones o fenómenos que tienen lugar en un espacio geográfico y que produjeron o están produciendo externalidades negativas tanto al medio físico como al social (GARCÍA, 1994).

Con acogimiento a los establecido por BASTERRECHEA et al. (1996) y OROPEZA, (1999), además de los niveles ya expuestos, son tres las características fundamentales de carácter hidrológico y sociopolítico que definen a las cuencas hidrográficas: a) líneas divisorias de agua como límites naturales totales o parciales; b) una porción de territorio drenada por un sistema de tributarios que contribuyen a alimentar un curso de agua principal, pudiendo verter a otra cuenca, un lago o el mar; c) en ellas se da una dinámica ambiental definida por las interacciones sistémicas entre el agua, el suelo y la vegetación, y el impacto que sobre éstos tienen las decisiones políticas y económicas de los seres humanos que en ella viven y de otros que habitan fuera de la cuenca.

CEPAL (2001) plantea que la cuenca debe verse como un sistema integrado, en el que el agua es un recurso compartido y que se administra parceladamente, por lo cual se crean situaciones de conflicto con relación a la gestión del agua. Para GWP (2000) y LARSEN et al. (2001), la integración, como tal, puede ser considerada bajo dos categorías básicas: como sistema natural o parte biofísica, con su importancia y situaciones críticas en cuanto a disponibilidad, cantidad y calidad; como sistema humano o socioeconómico, determinado por el uso del recurso, sus prioridades de desarrollo y su deterioro ambiental. 


\section{Cambio climático}

El sistema climático terrestre mantiene desde hace 600 millones de años su temperatura media dentro de un rango de variación pequeño, según MARTíN (1999). El Cambio Global en el que la Tierra siempre ha estado inmersa, inició un modificación de su trayectoria hace unos 200 años debido a las nuevas actividades antrópicas, proceso que se viene acelerando desde el pasado siglo y que adquiere mayor dimensión en el actual, situación que ha generado cambios drásticos en la superficie, en la atmósfera, en el uso de la tierra, en el agua continental, en el mar y en las zonas costeras, así como en la biodiversidad al eliminar o modificar ecosistemas, como lo señala FERNÁNDEZCARRASCO (2002).

Plantea ACACIA (1999) que se tienen incrementos de la temperatura de la superficie global de la tierra desde el siglo XIX. Por ejemplo, en Europa, durante el siglo $X X$, la temperatura media anual tuvo un aumento de $0,8^{\circ} \mathrm{C}$, siendo la última década (1990-1999) la más caliente registrada hasta el momento, tanto en las temperaturas medias anuales como las temperaturas invernales. Por otro lado, este mismo autor indica que las temperaturas nocturnas han aumentado más que las diurnas, probablemente debido al aumento de la nubosidad y que, por ejemplo, la precipitación sobre el norte de Europa ha aumentado entre un 10 y un $40 \%$, mientras que en algunas partes del sur de Europa la precipitación ha disminuido hasta un $20 \%$.

A nivel global, según IPCC (2001) y teniendo como referencia diferentes escenarios de emisiones, se estimó que para el año 2100, un incremento en la temperatura global media entre uno y cuatro grados por encima de la actual. Se advierte que no serán únicamente las temperaturas las que sufrirán cambios, los patrones de precipitación van a mudar considerablemente, dando lugar a que algunas zonas sean más húmedas y otras más secas de lo que son actualmente.

FERNÁNDEZ-CARRASCO (2002) señala que los efectos probables del cambio climático afectarán a los extremos meteorológicos, en algunas regiones se acentuarán las presiones futuras sobre los recursos hídricos y su gestión, se ocasionarán cambios en los ecosistemas naturales, pérdida de algunos hábitats críticos, mayor riesgo de sequías e incendios en las zonas tropicales, cambios en la fauna acuática y la biodiversidad de moluscos, así como reducción de los glaciares de montaña, dentro de los aspectos positivos se pueden incluir el aumento de la productividad neta de algunos ecosistemas temperados.

IPCC (2000) señala que los cambios en el uso del suelo, actividad de origen humano, que se ha incrementado sustancialmente en la última parte del siglo $X X$, han liberado grandes cantidades de carbono como consecuencia de la tala de bosques en todo el globo terráqueo. Es evidente que las modificaciones de la cubierta vegetal provocan cambios en la fracción de la radiación solar reflejada por la superficie terrestre, cambios en el balance dentro del ciclo del agua, que modifican las tasas de evapotranspiración así como las de humedad retenida por la vegetación, provocando cambios en la distribución y las características de las denominadas fuentes y sumideros de gases de efecto invernadero, con efectos tanto a escala local como global que se manifiestan en cambios climáticos y que se hacen evidentes en incremento o decremento, por ejemplo, del régimen de lluvias.

FERNÁNDEZ-CARRASCO (2002) sostiene que científicamente está demostrado que la salud humana, los ecosistemas y los sectores socioeconómicos, vitales para lograr un desarrollo sostenible, son sensibles a los cambios del clima y en particular a la magnitud y rapidez del cambio climático y sus variabilidades. El cambio climático puede suponer un importante factor adicional de deterioro de los sistemas ya afectados por una creciente demanda de recursos, por unas prácticas de gestión insostenibles y por la contaminación, que en muchos casos pueden ser de magnitud igual o mayor que el impacto debido al cambio climático.

\section{Cambio climático y recurso hídrico}

En la valoración de 1995 del Grupo de Intergubernamental de Expertos sobre los cambios Climáticos (IPCC, 1996) se afirmaba que el cambio climático originaría una intensificación del ciclo hidrológico global que tendría consecuencias importantes sobre los recursos hídricos regionales. En este caso se puede decir que los jagüeyes como un sistema ambiental hídrico y de tipo antrópico, con gran valor regional, también se verían involucrados.

Por su parte ACACIA (1999) determina en cuanto al componente hídrico, que las presiones actuales y futuras sobre los recursos hídricos y su gestión, se verán exacerbadas por el cambio climático, en parte porque el efecto del cambio es incierto. Probablemente aumentará el riesgo de inundaciones para algunas regiones, mientras que en otras habrá escasez de agua; igualmente de forma temporal podrán suceder en una misma región largos períodos de inundaciones y extensos períodos de sequía.

También de forma relacionada, ACACIA (1999) sostiene, que en las regiones de climas calientes y más secos, la calidad del suelo se verá deteriorada y esto tendrá un efecto negativo sobre las funciones ecosistémicas del suelo y sobre la sociedad. La magnitud de este efecto 
variará claramente entre regiones geográficas, y podría verse atenuada por un aumento de la precipitación, excepto en los casos en que cambios en la precipitación exacerben los procesos de degradación del suelo, situación en la que el recurso hídrico actuará como un tensor ambiental de magnitud negativa.

En cuanto tiene que ver con la productividad de los sistemas hídricos, ACACIA (1999), arguye, que el cambio climático probablemente resultará en cambios que afectarán a la fauna acuática tanto marina como de agua dulce y a la biodiversidad en ellos contenida o de ella dependiente. Los cambios en la industria piscícola y pesquera serán agravados por unos niveles de explotación insostenibles y por los cambios ambientales que se producirán.

FERNÁNDEZ-CARRASCO (2002) conceptúa que la demanda de agua para los usos agrícolas, de abastecimiento a poblaciones y energéticos se verá modificada. El sector agrario modificará su demanda en el mismo sentido en el que se modifique el déficit hídrico del suelo. Los aprovechamientos eléctricos pueden ser afectados por una pérdida parcial del potencial hidroeléctrico bruto y la concentración de la producción en periodos de tiempo más cortos. Las prioridades entre los distintos usos también pueden verse afectadas, al ser distinto el volumen de agua disponible. Igualmente, expresa que la mayoría de los países en desarrollo son muy vulnerables al cambio climático, ya que muchos de ellos están situados en regiones áridas y semiáridas y en su mayoría, obtienen sus recursos hídricos de puntos de abastecimiento únicos, como por ejemplo, perforaciones o embalses aislados.

Los sistema hídricos rurales - como los jagüeyes del Caribe colombiano (Figura 1) - e incluso urbanos de muchas zonas de los países en vías de desarrollo, son sistemas de suministro que por su naturaleza son muy vulnerables; estos lugares, en general carecen de reservas alternativas en caso de necesidad. Además, dada la escasez de recursos técnicos, financieros y de gestión, adecuarse a las situaciones de escasez e implementar medidas de adaptación representa una pesada carga para sus economías (FERNÁNDEZ-CARRASCO, 2002).

De acuerdo con lo establecido en http://www. cambioclimatico.org/content/recursos-hidricos, el cambio en las pautas de precipitaciones afectará la cantidad de agua que se capta. Muchos modelos climáticos indican que los aguaceros en general se volverán más intensos. Esto incrementará las escorrentías e inundaciones y reducirá al mismo tiempo la capacidad del agua para infiltrarse en el suelo. El cambio en las pautas estacionales puede afectar la distribución regional de los suministros de agua subterránea y superficial.
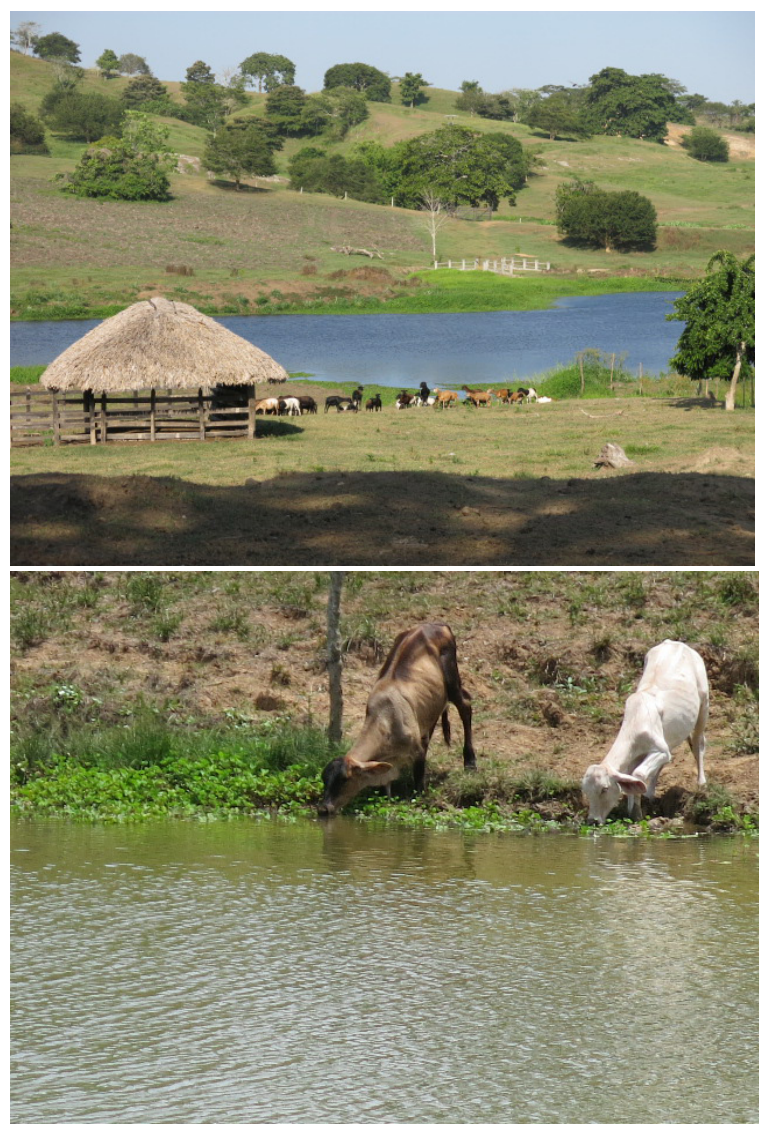

Figura 1. Jagüeyes característicos de la zona rural del municipio de Sincelejo, Sucre, Colombia y su uso pecuario. Fotos: De La Ossa, V.J.

En el plano local, la vegetación y las propiedades físicas de las zonas de captación influirán aún más en la cantidad de agua que se retenga. Señalan, al mismo tiempo, que cuanto más seco sea el clima, más sensible será la hidrología local; en los climas secos, cambios relativamente pequeños de la temperatura y las precipitaciones pueden causar cambios relativamente grandes en las escorrentías. Por consiguiente, las regiones áridas y semiáridas serán particularmente sensibles a la disminución de las lluvias y a la mayor evaporación y transpiración de las plantas. Muchos modelos climáticos proyectan una reducción de las precipitaciones en las regiones ya secas de Asia central, el Mediterráneo, África meridional y Australia, por ejemplo (http://www.cambioclimatico.org/content/ recursos-hidricos).

MIRALLES-WILHELM (2014) expresa respecto del suministro, distribución y sostenibilidad de fuentes de agua. que la característica de mayor relevancia para la mayoría de los países en el Caribe, es la escasez de agua y/o acceso restringido, donde la demanda es igual o mayor al suministro de la 
misma, mientras continúa aumentando la demanda gracias al crecimiento de la población y su desarrollo. Una situación parecida se encuentra en muchas áreas de países más grandes como México, Brasil, Chile y Perú. Hasta en países con amplios recursos hídricos para abastecer su población, la distribución del agua se lleva a cabo de una manera insostenible e inconsistente en la mayoría de los casos, resultando en una mayor amenaza a las fuentes de agua debida a los impactos del cambio climático.

\section{Riqueza hídrica colombiana}

Tomando como base el marco político para la gestión integral del recurso hídrico (PNGIRH) (MAVDT, 2010), se tiene que por su localización geográfica, su orografía y una gran variedad de regímenes climáticos, Colombia se ubica entre los países con mayor riqueza en recursos hídricos en el mundo. Sin embargo, cuando se considera en detalle que la población y las actividades socioeconómicas se ubican en regiones con baja oferta hídrica, que existen necesidades hídricas insatisfechas de los ecosistemas y que cada vez es mayor el número de impactos de origen antrópico sobre el agua, se concluye que la disponibilidad del recurso es cada vez menor y que para muchas regiones es crítica.

Según estimaciones del IDEAM (2005), en promedio en Colombia la precipitación media anual es de 3.000 $\mathrm{mm}$ con una evapotranspiración real de $1.180 \mathrm{~mm}$ y una escorrentía medial anual de $1.830 \mathrm{~mm}$. Teniendo en cuenta lo anterior, del volumen de precipitación anual, $61 \%$ se convierte en escorrentía superficial generando un caudal medio de $67.000 \mathrm{~m}^{3} / \mathrm{seg}$, equivalente a un volumen anual de $2.084 \mathrm{~km}^{3}$ que escurren por las cinco grandes regiones hidrológicas que caracterizan el territorio nacional continental, de la siguiente forma: $11 \%$ en la región Magdalena - Cauca, 5\% en la región del Caribe; $18 \%$ para la región del Pacífico; $34 \%$ en la región de la Amazonia y $32 \%$ en la región de la Orinoquia.

Estimaciones realizadas por el IDEAM en sus diferentes estudios, anotan que la escorrentía superficial per cápita total del país es de $57.000 \mathrm{~m}^{3}$ al año, en cuanto a la oferta neta en la cual se incorporan reducciones tanto por alteración de la calidad como por regulación natural, se alcanzan apenas los $1.260 \mathrm{~km}^{3}$ que corresponden a una disponibilidad de $34.000 \mathrm{~m}^{3} /$ persona/año. En las condiciones de año seco consideradas, esta disponibilidad se reduce a 26.700 metros cúbicos por persona al año.

La riqueza hídrica colombiana también se manifiesta en la favorable condición de almacenamiento superficial, representada por la existencia de cuerpos de agua lénticos, distribuidos en buena parte de la superficie total y por la presencia de enormes extensiones de ecosistemas de humedales. Del volumen total de escorrentía anual $1,81 \%$ se almacena superficial y temporalmente de la siguiente manera: $0,47 \%$ en pantanos, $1,30 \%$ en lagos naturales y $0,04 \%$ en los páramos, constituyéndose en la oferta de almacenamiento ambiental que bajo ciertas condiciones racionales es utilizada, bien sea para otros usos productivos o para el funcionamiento de los sistemas naturales (IDEAM, 2005).

De otro lado, es necesario destacar que la distribución heterogénea de la oferta de agua, de la población y de las actividades económicas en las diferentes regiones del país, hacen que la relación oferta - demanda sea menos favorable en aquellas zonas donde los rendimientos hídricos son menores y mayores las concentraciones de demanda. Esta situación unida a que más del $80 \%$ de los asentamientos urbanos de los municipios se abastecen de fuentes muy pequeñas, de baja capacidad de regulación como: arroyos, quebradas, riachuelos - incluso jagüeyes - con limitados o nulos sistemas de almacenamiento, hacen que una buena parte de la población y su abastecimiento de agua sea altamente vulnerable.

En cuanto a la demanda, en estimaciones realizadas por el IDEAM, la demanda para el desarrollo de las actividades socioeconómicas en Colombia se representa principalmente mediante los siguientes usos: agrícola, doméstico, industrial, pecuario y servicios; el uso que presenta un mayor porcentaje es el agrícola con el $54 \%$, con el $29 \%$ el doméstico y con el $13 \%$ el industrial, en menor escala el pecuario y el de servicios con porcentajes del $3 \%$, y el $1 \%$, respectivamente.

Cuando se presenta el fenómeno de El Niño hay déficit moderado de precipitación (entre el 20 y el $40 \%$ en los volúmenes mensuales) en la región Caribe y la mayor parte de la región Andina, particularmente en Nariño, Valle, norte del Huila, occidente de Antioquia, Tolima, Cundinamarca, Boyacá, Santander y la región del Catatumbo. Estas deficiencias son de carácter severo (superiores al 40\%) en La Guajira, la parte media del litoral Caribe, el norte de Córdoba, el sector central de Sucre, el altiplano Cundiboyacense y el área limítrofe entre los Santanderes, como se menciona en los informes de evaluación del fenómeno de El Niño del IDEAM (IDEAM, 2002).

\section{Los jagüeyes como unidades hídricas}

La definición de jagüey, según se establece en América es: balsa, pozo o zanja llena de agua, ya artificialmente, ya por filtraciones naturales del terreno. RAE (Real Academia de la Lengua Española), dice al respecto que en Colombia se utiliza el término jagüey para 
depósitos superficiales de agua en zonas con sequías estacionales prolongadas; posiblemente el término esté relacionado con la cultura Wayúu (guajiros). Al igual que la voz Jagüel, usada en el Cono Sur para indicar un pozo o zanja que retiene el agua de lluvia y sirve para abrevar el ganado o el riego. Proviene de la palabra quichua jagüei o jagüey (Figura 2).

Los jagüeyes, también conocidos como aguajes, ollas de agua, cajas de agua, aljibes, pozos, trampas de agua o bordos de agua, son depresiones sobre el terreno, que permiten almacenar agua proveniente de escurrimientos superficiales. Jagüey es un vocablo taíno que significa balsa, zanja o pozo lleno de agua, en el que abreva el ganado (SAGARPA, 2009), se le clasifica como cuerpos de agua epicontinentales (BOTERO et al., 2009).

Se define, así mismo, un jagüey como un cuerpo de agua más pequeño que un lago o una presa, aunque no hay un rango de tamaño bien definido, puede ser desde menos de una ha hasta 10 has. No obstante, en el Reino Unido, donde se tienen algunos de los estudios más extensos sobre bordería multipropósito se ha adoptado una extensión para este tipo de obras de entre varios $m^{2}$ y 2 ha, siempre que sobre estas se retenga agua por al menos cuatro meses al año. Los jagüeyes son un caso particular de la captación de aguas lluvias, misma que ha sido conocida en México y Centro América desde las épocas prehispánicas, tal como lo demuestra la construcción de "Chultuns" en la región Maya (SAGARPA, 2009).

Según CERVANTES (1994) los jagüeyes son lagunas con aparente similitud con los lagos, su existencia puede corresponder a cualquier origen, drenaje y dimensiones. Igualmente, indica que permanecen relativamente estancados y son inestables, con variaciones en el nivel de agua; pueden ser temporales o permanentes, dependiendo del régimen pluvial.

Históricamente SEMARNAT (2009) referenciando a México, señala que en lo que respecta a los depósitos pluviales a cielo abierto se destacan los jagüeyes, que fueron muy comunes en el centro y el sur de México, en especial en las zonas áridas y semiáridas donde el nivel freático estaba muy bajo o el suelo era rocoso y resultaba muy difícil alcanzarlo mediante la excavación de pozos someros. Alos jagüeyes, hechos artificialmente o acondicionados aprovechando hondonadas naturales, situados en terrenos cercanos a cerros y lomeríos, se canalizaba el agua de las pequeñas corrientes pluviales o de los escurrimientos de los cerros y techos aledaños.
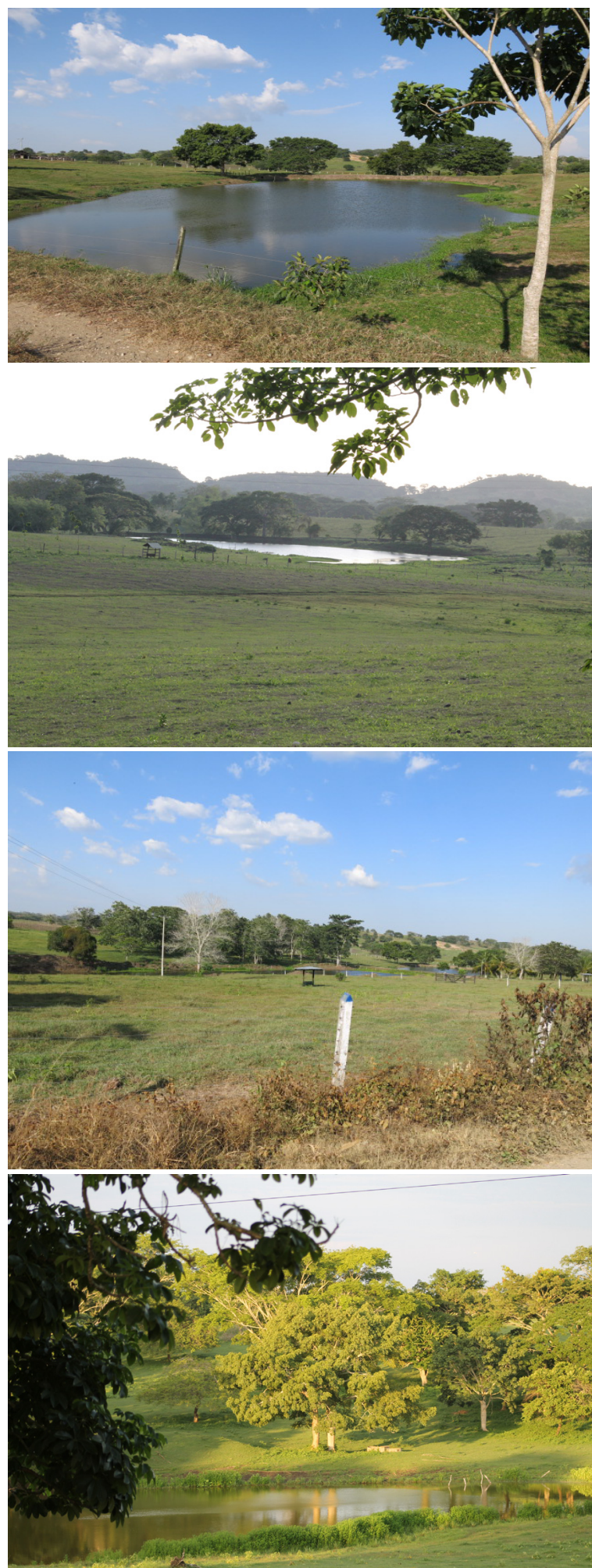

Figura 2. Jagüeyes artificiales del Caribe colombiano. Fotos: De La Ossa, V.J. 
En cuanto a Colombia, RAS (2010) hace referencia, indicando que en la región Caribe colombiana, pero especialmente en la alta Guajira donde la oferta hídrica es muy baja, existen muchos cauces secos que se convierten en arroyos cuando se producen lluvias intensas. Pasada la temporada de lluvias en estos cauces secos quedan ciertos sitios con charcos de agua que en el lenguaje local se conocen como jagüeyes. Éstos son unos reservorios naturales de agua, pero también pueden ser construidos mediante excavación y conformación de diques o terraplenes aprovechando ondulaciones del terreno para captar y almacenar aguas Iluvias por escorrentía. Tradicionalmente han sido construidos por ganaderos como abrevaderos para los animales, pero allí también concurren personas para proveerse de agua con los consiguientes riesgos que implica su consumo sin tratamiento previo (BOTERO et al., 2009).

DECLERK et al. (2006) demuestran que existe una relación positiva entre el número de jagüeyes y la biodiversidad de un área dada; las áreas acuáticas restringidas como los jagüeyes juegan un importante papel en la conservación, contribuyen significativamente al mantenimiento y conservación de la biodiversidad regional, en especial cuando poseen como habitantes especies raras, endémicas o únicas, por esta razón la creación de nuevas unidades viene siendo usada como estrategia ampliamente practicada en muchos países. CHARÁ et al. (2008) indican que permiten, en efecto, el establecimiento y conservación de especies vegetales y organismos animales diversos, proveen alimento y refugio, tanto para seres vivos propios del agua como de aquellos que se asocian al sistema por las facilidades ofrecidas. Para la región Caribe en especial para el departamento de Sucre se establece que los jagüeyes son de gran importancia ambiental, actúan como hábitat o refugio de muchas especies locales, por ejemplo de avifauna local y migratoria (DE LA OSSA et al., 2012), Crocodílideos (DE LA OSSA y DE LA OSSA-LACAYO, 2013; DE LA OSSA-LACAYO, 2014) y quelonios (DE LA OSSA et al., 2016 en prep.), entre otros taxones (BOTERO et al., 2009) (Figura 3).

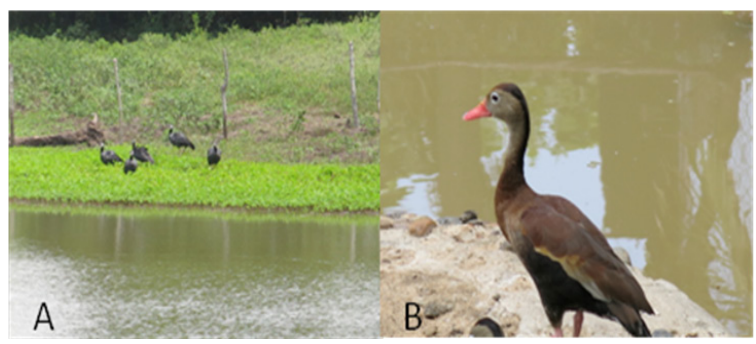

Figura 3. Jagüeyes regionales con presencia de Chauna chavaria (chavarrí) (A) y Dendrocygna autumnalis (pisingo)

(B). Fotos: De La Ossa, V.J.

\section{Referencias}

ACACIA 1999. Valoración de los efectos potenciales del cambio climático en Europa. Informe ACACIA (borrador). M. Parry, C.Parry y M. Livermore (Eds). Disponible en: http://ecce.uclm.es/documentos/publicos/ACACIA resumen\%20 ES.pdf. Consultado: 05-06-2015.

AGUILAR-ROBLEDO, M. 1995. Autopsia de un fracaso: El caso del Proyecto Pujal-Coy de la Huasteca Potosina. Editorial Ponciano Arriaga. Gobierno del Estado de San Luis Potosí, México.

ALBÁN, J.; CARVAJAL, M.; DOMíNGUEZ, J.; JUMBO, C. 2003. Gestión Pública de los Recursos Naturales. Instituto de Estudios Ecuatorianos-Fraga Impresores. Quito, Ecuador.

ALBUQUERQUE, G. 2000. Gestión de Cuencas: Agua, Gente y Ambiente. Págs. 179-189. En: // Curso Internacional de Aspectos Geológicos de Protección Ambiental. UNESCO.

BALLUT-DAJUD, G.; MONROY-PINEDA, M.C. 2015. Los jagüeyes del municipio de Sincelejo, Sucre, Colombia. Rev Colombiana Cienc Anim. 7 (1):80-83.

BASTERRECHEA, M.; DOUROJEANNI, A.; GARCÍA, L.; NOVARA, J.; RODRÍGUEZ, R. 1996. Lineamientos para la preparación de proyectos de manejo de cuencas hidrográficas para eventual financiamiento del Banco Interamericano de Desarrollo. Washington. D.C.

BOTERO, A.L.; DE LA OSSA, V.J.; ESPITIA, P.A.; DE LA OSSA-LACAYO, A. 2009. Importancia de los jagüeyes en las sabanas del Caribe colombiano. Rev. col. Cien. Anim. 1 (1):71-84. 
CASTELÁN, E. 2000. Los Consejos de Cuenca en México. Págs. 175-187. En: Asignación, productividad y manejo de recursos hídricos en cuencas. Instituto Internacional del Manejo del Agua (IWMI), Serie Latinoamericana, No. 20. México.

CASTIBLANCO, R.C. 2006. Manual de valoración económica del medio ambiente. Instituto de Estudios Ambientales IDEA, Universidad Nacional de Colombia. Bogotá.

CEPAL-Red de Cooperación en la Gestión Integral de Recursos Hídricos para el Desarrollo Sustentable en América Latina y el Caribe. 2001. Carta Circular No. 15. Disponible en: http://www.cepal.org/es/publicaciones/36569-cartacircular-la-red-cooperacion-la-gestion-integral-recursos-hidricos. Consultada: 12-07-2015.

CERVANTES, M. 1994 (Compilador). Guía regional para el conocimiento, manejo y utilización de los humedales del noroeste de México. ITESM-Campus Guaymas/CECARENA, México: 27-82.

CHARÁ, J.; PEDRAZA, O.G.; GIRALDO, S.L. 2008. Ganadería del futuro: Investigación para el desarrollo. Corredores ribereños como herramienta de protección de ambientes acuáticos en zonas ganaderas, Cap. 5. CIPAV, Cali, Colombia: $111-129$

COLBY, M. 1990. Environmental Management in Development: The Evolution of Paradigms. Ecological Economics 3:193-213

DANILO, A.; DÍAZ C. 2000. Sequía en un mundo de agua. CIRA-UAEM. Disponible en: http://tierra.rediris.es/hidrored/ ebooks/sequia/pdf/00intros.pdf. Consultada: 10-07-2015.

DE LA OSSA, V.J.; GALVÁN-GUEVARA, S.; DE LA OSSA-LACAYO, A. 2102. Importancia del jagüey ganadero en la conservación local de aves silvestres en el Caribe colombiano. Rev. U.D.C.A Act. \& Div. Cient. 15 (1):181 - 186.

DE LA OSSA, V.J.; ARDILA-MARULANDA, M.; DE LA OSSA-LACAYO, A. (en prep). Quelonios y jagüeyes del golfo de Morrosquillo, Caribe colombiano

DE LA OSSA V.J.; DE LA OSSA-LACAYO, A. 2013. Ocupación de jagüeyes por la babilla, Caiman crocodilus fuscus (Cope, 1868), en el Caribe colombiano. Biota Colombiana 14 (2): 327-336.

DE LA OSSA-LACAYO, A. 2014. Jagüeyes: ecosistemas lénticos y antrópicos como alternativa para la conservación de Caiman crocodilus fuscus (Crocodylia : Alligatoridae) en el golfo de Morrosquillo, Sucre, Colombia. Rev. Asoc. Col. Cienc. Biol. 26:21-28.

DECLERCK, S.; DE BIE, T.; ERCKEN, D.; HAMPEL, H.; SCHRIJVERS, S.; VAN WICHELEN, J.; GILLARD, V.; MANDIKI, R.; LOSSON, B.; BAUWENS, D.; KEIJERS, S.; VYVERMAN, W.; GODDEERIS, B.; DE MEESTER, L.; BRENDONCK, L.; MARTENS, K. 2006. Ecological characteristics of small farmland ponds: Associations with land use practices at multiple spatial scales. Biological Conservation 131 (4):523-532.

DOUROJEANNI, A. 2002. Gestión del agua a nivel de cuencas: teoría y práctica. Serie: Recursos naturales e infraestructura. CEPAL. Santiago de Chile.

DOWNING, J. A.; PRAIRIE, Y.T.; COLE, J.J.; DUARTE, C.M.; TRANVIK, L.J.; STRIEGL, R.G.; MCDOWELL, W.H.; KORTELAINEN, P.; CARACO, N.F.; MELACK. J.M.; MIDDELBURG, J . 2006. The global abundance and size distribution of lakes, ponds, and impoundments. Limnology and Oceanography 51:2388-2397.

ENVIRONMENTAL RESOURSES MANAGEMENT Y DEPARTAMENT FOR INTERNATIONAL DEVELOPMENT.1997. Economía Ambiental y su Aplicación a la Gestión de Cuencas Hidrográficas. 2 edición. Ministerio de Agricultura. Santiago de Chile.

FERNÁNDEZ-CARRASCO, P. 2002. Estudio del impacto del cambio climático sobre los recursos hídricos. Aplicación en diecinueve pequeñas cuencas en España. Tesis Doctoral. Universidad Politécnica de Madrid. Departamento de Matemática e Informática Aplicada a la Ingeniería Civil. E.T.S.I. Caminos, Canales y Puertos. España. 
GALLOPÍN, G. 1985. Tecnología y sistemas ecológicos. Boletín de Medio Ambiente y Boletín de Medio Ambiente y Urbanización 3 (12):1-16.

GALLOPÍN, G. 2000. Ecología y Ambiente. Págs. 88-137. En: Leff, E. (Coord.). Los problemas del conocimiento y la perspectiva ambiental del desarrollo. Siglo Veintiuno Editores. México.

GARCÍA, R. 1986. Conceptos básicos para el estudio de sistemas complejos”, en Leff, E. (Coordinador), Los problemas del conocimiento y la perspectiva ambiental del desarrollo, México, Siglo XXI, pp. 381-409.

GARCÍA, R. 1994. Interdisciplinariedad y sistemas complejos. Págs. 85-124. En: Leff, E. (Coord.). Ciencias Sociales y formación ambiental. Gedisa-UNAM. México.

GRIGG, N. 1996. Water Resources Management, Principles, Regulations and Cases. McGraw-Hill. USA.

GWP - Global Water Partnership. 2000. Hacia la Seguridad Hídrica: un Marco de Acción. Resumen Ejecutivo. Reino Unido. .

IDEAM - Instituto de Hidrología, Meteorología y Estudios Ambientales. 2002. Efectos naturales y socioeconómicos del fenómeno El Niño en Colombia. Bogotá D.C.

IDEAM - Instituto de Hidrología, Meteorología y Estudios Ambientales. 2005. El Estudio Nacional del Agua un Compendio sobre el Recurso hídrico en Colombia. Bogotá D.C., 2005.

IPCC, 1996. Houghton, J.T.; Meira Filho, R.; Callander, B.A.; Harris, N.; Kattenberg, A.; Maskeli, K. (eds.). En: Climate Change 1995. The Science of Climate Change. Cambridge University Press. Cambridge.

IPCC, 2000. Tercer Informe de Evaluación del IPCC. Informe Especial sobre escenarios de Emisiones del IPCC. Disponible en: https://www.ipcc.ch/pdf/special-reports/spm/sres-sp.pdf. Consultado: 21-06-2016.

IPCC, 2001. Climate Change 2001: Impacts, Adaptation, and Vulnerability. A report of Working Group II of IPCC. Disponible en: https://www.ipcc.ch/ipccreports/tar/wg2/. Consultado: 23-06-2016.

KALFF, J. 2001. Limnology: Inland water ecosystems. Prentice Hall. USA.

LARSEN, H. 2001. The Application of Models in Integrated River Basin Management. Dsiponible en: http://www. dhisoftware.com/mikebasin/Publications/MikeBasinIWRM.pdf. Consultado: 05-11-2016.

MARTíN, J. 1999. Cambios Climáticos. Una aproximación al sistema Tierra. Ediciones libertarias. Madrid.

MARTínEZ, M. 1999. Manejo Integral de Cuencas. Pasado, Presente y Futuro. Pág. 1-11. En: Simposio Manejo Integral de cuencas hidrográficas del IX Congreso Nacional de Irrigación. ANEI. Culiacán, Sinaloa, México.

MAVDT - Ministerio de Ambiente, Vivienda y Desarrollo Territorial. 2010. Política nacional para la gestión integral del recurso hídrico. Viceministerio de Ambiente. Dirección de Ecosistemas. Grupo de Recurso Hídrico. Bogotá, Colombia.

MELVILLE, R. 1996. Política Hidráulica Mexicana: Oportunidades para la investigación. Págs. 17-29. En: Apropiación y usos del agua, nuevas líneas de investigación. Universidad Autónoma Chapingo. Chapingo, México.

MELVILLE, R. 2000. La cuenca fluvial, como territorio fragmentado para la organización del aprovechamiento, conservación y administración de los recursos hídricos. Págs. 57-77. En: Asignación, productividad y manejo de recursos hídricos en cuencas. Instituto Internacional del Manejo del Agua (IWMI), Serie Latinoamericana No. 20. México.

MEYBECK, M. 1995. Global distribution of lakes. Págs.. 1-35. En: Lerman, A.; Imboden, D.M.; Gat, J.R. (Eds). Physics and chemistry of lakes. Springer-Verlag. 
MIRALLES-WILHELM, F.R. 2014. Recursos hídricos y adaptación al cambio climático en Latinoamérica y el Caribe. Directrices estratégicas y líneas de acción propuestas. Banco Interamericano de Desarrollo. Nota técnica 478 . USA.

MIRAMONTES, O. 1999. Los sistemas complejos como instrumentos de conocimiento y transformación del mundo. Págs. 83-92. En: Ramírez, S. (Ed.). Perspectivas en las teorías de sistemas. Siglo XXI. UNAM. México.

MONTES, J.; LEFF, E. 2000. Perspectiva ambiental del desarrollo del conocimiento.Págs. 1-26. En: Leff, E. (Coord.). Los problemas del conocimiento y la perspectiva ambiental del desarrollo. Siglo Veintiuno Editores. México.

OPS/OMS, 2001. Agua y Salud: un brindis por la vida. "PS/OMS. Lima, Perú.

OROPEZA, J. 1999. Modelos matemáticos y su aplicación al manejo de cuencas y Paradigmas. En IX Congreso Nacional de Irrigación. Simposio 4. Manejo Integral de Cuencas Hidrológicas. ANEI, A.C. México.

PATRICK, R. 1994. Is Water Our Next Crisis?. Proceedings of the American Philosophical Society 138 (3):371-376.

QUINTANA, S.; FUNTOWICZ, S. 1998. Cómo afrontar la problemática compleja del agua. Págs. 1-10. En: Simposio Gestión de aguas, participación ciudadana y conflictos sociales y políticos del Congreso sobre Planificación y Gestión de Aguas. España.

RAS - Reglamento Técnico del Sector de Agua Potable y Saneamiento Básico. 2010. Título J. Alternativas Tecnológicas en Agua y Saneamiento para el Sector Rural. Ministerio de Ambiente, Vivienda y Desarrollo Territorial Viceministerio de Agua y Saneamiento. Bogotá, Colombia.

RODRÍGUEZ M.; ESPINOZA G. 2003. Gestión ambiental en América Latina y el Caribe: Evolución, tendencias y principales prácticas. Banco Interamericano de Desarrollo. USA.

SAGARPA - Secretaría de Agricultura, Ganadería, Desarrollo Rural, Pesca y Alimentación. 2009. Ollas de agua, Jagüeyes, Cajas de agua o Aljibes. Subsecretaría de Desarrollo Rural. Dirección General de Apoyos para el Desarrollo Rural. México.

SANTACRUZ-DE LEÓN, G. 2007. Hacia una gestión integral de los recursos hídricos en la cuenca del río Valles, Huasteca, México. Tesis de Doctorado. Universidad Autónoma de San Luis Potosí. Facultades de Ciencias Químicas, Ingeniería y Medicina. Programa Multidisciplinario de Posgrado en Ciencias Ambientales. México.

SCHUILING, R. D. 1977. Source and composition of lake sediments. Págs. 12-18. En: Golterman, H.L. (Ed.). Interaction between sediments and fresh water. Proceedings of an international symposium held at Amsterdam. Netherlands.

SEMARNAT. 2009. Semblanza Histórica del Agua en México. Comisión Nacional del Agua. Secretaría de Medio Ambiente y Recursos Naturales. México.

SHIKLOMANOV, I.A.; RODDA, J.C. 2003. World water resources at the beginning of the twenty-first century. Cambridge Univ. Press. UK.

WETZEL, R.W. 1990. Land-water interfaces: Metabolic and limnological regulators. Int. Verein. Theor. Limnol. Verh. 24:6-24. 